\title{
IL SISTEMA DI GESTIONE PER LA QUALITÀ SECONDO LA NORMA ISO900 I:2008
}

\section{UN TEMA DI GRANDE ATTUALITÀ NEL MONDO INDUSTRIALE E SANITARIO.}

"Va dove ti porta il cuore" è un messaggio affascinante per ogni uomo.

"Va dove ti porta il cliente" è l'indicazione obbligatoria per una impresa oazienda sanitaria che intenda giocare bene la propria sfida per il futuro. Come risultato di profondi cambiamenti sociali e culturali, nel mondo dei consumi e dei servizi i clienti (aziende, utenti, singoli consumatori, pazienti), hanno esigenze di beni e servizi sempre diversi e di complessità crescente.

C'è però una componente che rimane costante: $\mathrm{i}$ clienti, oggi più consapevoli, chiedono istintivamente che beni e servizi abbiano "qualità".

Il concetto di qualità porta in se una grossa carica di indeterminatezza; si può in linea di massima condividere il pensiero che, per un cliente, un bene o servizio è di qualità quando questo soddisfa le sue esigenze.

Nel settore della salute, che i processi diagnostici o terapeutici abbiano il minimo possibile di indeterminatezza e di errore.

Come logica conseguenza, le strutture che possono offrire una garanzia di qualità dei propri prodotti e servizi, qualità intesa come piena soddisfazione del cliente, hanno dei vantaggi competitivi. C'è una via maestra internazionale aperta, nel mondo produttivo e dei servizi, per poter offrire ai clienti la garanzia della qualità di cui stiamo parlando: la strada è quella di far certificare il Sistema di Gestione per la Qualità (SGQ) aziendale secondo la Norma ISO 9001:2008.

Protagonista della certificazione è quindi la Qualità in tutta la struttura. Oppure - quanto meno - nei servizi nei quali la sensibilità alla qualità è superiore a quello dell'intera organizzazione.

Tentiamo di capire meglio, approfondendo il significato delle parole sottolineate:

\section{Norma IS0 9001:2008}

È stata redatta da un organismo internazionale, ed è riconosciuta in tutto il mondo industrialmente avanzato. Prescrive i requisiti minimi di Qualità che in una azienda o in un servizio debbono essere garantiti dalla direzione, dall'area commerciale, dalla ricerca e sviluppo, dagli approvvigionamenti, dalla produzione, dal controllo qualità, dalla movimentazione e spedizione, dall'assistenza post vendita, cioè in pratica da tutta l'azienda. Nata per il mondo industriale, la Norma ISO fin dalla sua prima versione (1987) è stata decodificata per l'applicazione nel mondo sanitario.

2 Sistema di Gestione per la Qualità (SGQ) È la struttura organizzativa, le responsabilità, le procedure e le risorse messe in atto per la conduzione aziendale per la Qualità.

Il SGQ è costituito, dai pensieri, dalla parole, dalle opere (senza omissioni), che l'azienda ha messo in essere per rispondere alle richieste della Norma ISO 9001:2008.

\section{Certificazione}

Un ente esterno accreditato (cioè competente e neutrale) certifica che il SGQ dell'azienda soddisfa le richieste della Norma.

È ragionevole concludere che i prodotti/servizi realizzati da una azienda o struttura sanitaria certificata siano di qualità, ed il cliente-paziente si possa fidare.

L'intendimento è quello di rassicurare sia il consumatore-cliente-paziente che il fornitore-produttore : il consumatore sa che, se un prodotto circola liberamente o un servizio è secondo Norma ISO, esso ottempera a certi requisiti essenziali che lo tutelano, il paziente sa che una struttura che ha standardizzato i processi di servizio possiede un sistema per ridurre il più possibile gli eventi avversi che possono sempre accadere; il produttore sa che, rispettando certe regole, troverà minori ostacoli alla commercializzazione dei propri prodotti.

Quanto scritto sopra dimostra che la via della certificazione, comunque volontaria, è in realtà un passaggio obbligato per ogni tipo di Azienda, anche in sanità, per poter dare evidenza che $i$ prodotti e i servizi sanitari offerti sono sotto controllo. Non solo perchè seguono le prescrizioni della Norma ISO ma anche perché il seguire la Norma rende trasparente l'organizzazione, standardizza $\mathrm{i}$

processi di servizio e fa condividere a tutti i componenti dell'organizzazione le metodologie operative. Costruire un SGQ aiuta a fare squadra, a

Corresponding author: Beppe Carugo

Responsabile Divisione Qualità e Formazione - MZ Congressi

Via Farini 8I - 20159 Milano - Tel 348-8979002

Beppe.carugo@mzcongressi.com

beppecarugo@virgilio.it 
definire le responsabilità, $a$ individuare $\mathrm{i}$ momenti di miglioramento organizzativo.

Costruire il SGQ rappresenta perciò un cambiamento culturale importante, che induce nel tempo ogni operatore ad una sempre più viva sensibilità nella ricerca del miglioramento continuo, vero motore della Norma ISO.

Negli anni anche lo Stato, attraverso le indicazioni del Ministero della Salute, ha emanato delle Norme per l'ACCREDITAMENTO ISTITUZIONALE, norme che sono di fatto fotocopia della Norma ISO e che autorizzano, se rispettate, all'esercizio della attività sanitaria e ai rimborsi regionali (in pratica sostituiscono, ma sono più prescrittive, il vecchio sistema di convenzionamento).

Non tutte le regioni hanno dato seguito al DPR 14/1/1997 che dava il via all'Accreditamento Istituzionale: tuttavia chi costruisce un SGQ secondo la Norma ISO9001:2000 è più che in regola con le prescrizioni del DPR.

Descriviamo in pochissime frasi il lavoro che porta alla certificazione.

La struttura che vuole costruire il proprio SGQ deve fare un'attenta e approfondita analisi dei propri processi di servizio e descriverli in una serie di documenti (Procedure Generali o Istruzioni Operative) che devono essere condivise da tutta l'organizzazione.

Deve esistere nella struttura una figura, il Responsabile Gestione della Qualità (RGQ), che ha il compito di tirare le fila nella realizzazione del SGQ e sintetizzare l'organizzazione in un "Manuale della Qualità", in cui mette in evidenza come l'operatività aziendale soddisfi le richieste della Norma ISO9001:2008.

Tramite le funzioni operative, l'azienda scrive le procedure, vincolanti per tutti, che prescrivono come svolgere le attività che hanno impatto sulla Qualità dei beni/servizi forniti, in coerenza con le linee guida descritte nel Manuale della Qualità.

Il manuale della Qualità è poi consegnato ad un Ente di Certificazione, che deve giudicare se il SGQ della struttura - così come descritto nel Manuale stesso - è conforme alla norma.

L'Ente di certificazione verifica successivamente - nel corso di una visita ispettiva della durata di uno-due giorni e che coinvolge tutte le funzioni interessate - che le attività operative del servizio vengono realizzate in conformità a quanto descritto nel Manuale Qualità e nelle Procedure.

Se il parere è positivo, viene rilasciata la certificazione ISO9001:2008 per il SGQ.

Annualmente vengono effettuate, a cura dell'Ente stesso, verifiche di sorveglianza e dopo tre anni si procede alla visita di rinnovo della certificazione. Compito dell'organizzazio- ne - ed in particolare del RGQ e della Direzione - è di tener vivo e applicato il SGQ.

Per costruire un SGQ occorre un tempo relativamente lungo, ma soprattutto occorre una tempificazione delle varie fasi che necessita di una assistenza esterna che ha la funzione sia di supporto che di "pungolo".

Per dare un'idea di questo timing, ecco quello definito per una struttura sanitaria:

I punti salienti di una realizzazione di un SGQ sono:

a una formazione iniziale e continua atta coinvolgere TUTTE le figure professionali dell'organizzazione

b il coinvolgimento di TUTTI nell'analisi dei processi e nella produzione dei documenti, ognuno secondo le proprie responsabilità (definite a priori).

c la continua e puntuale rilevazione degli eventi negativi che occorrono nell'organizzazione (definite in gergo "non conformità"), motore principale del miglioramento continuo dell'organizzazione stessa. Il principio di fondo è che rilevare le cose che non vanno è il primo passo per migliorare i servizi.

d l'individuazione degli INDICATORI DI PROCESSO, al fine di misurare costantemente la qualità dei servizi offerti per poterli di conseguenza migliorare.

Come disse Berwick, misurare senza cambiare è uno spreco. Cambiare senza misurare è scervellato...

Ma, prima di tutto, occorre una forte volontà da parte della Direzione di intraprendere la strada della qualità. Senza questa spinta propulsiva un percorso così complesso non avrebbe possibilità di successo.

Tuttavia le strutture sanitarie con un SGQ certificato oggi sono numerose, e in particolare numerosi sono i servizi (Laboratori, Anatomia Patologiche, Radiologie, R.S.A., Centri Trasfusionali). I Clinici hanno meno confidenza con la standardizzazione dei processi di cura, perché ritengono che la professionalità non possa essere massimizzata.

È questo un errore di prospettiva: il SGQ non annulla la capacità professionale, ma la riconduce entro i binari di un corretto

servizio al cliente-paziente, responsabilizzando tutta l'equipe all'individuazione dei migliori meccanismi per la riduzione degli errori (attraverso un monitoraggio continuo delle potenziali cause): il SGQ rappresenta per il clinico lo "zoccolo duro" organizzativo su cui innestare le proprie capacità professionali.

Ovvero, il SGQ è un efficace strumento di lavoro che, al pari dell'analizzatore, del fonendoscopio e 
del bisturi, necessita prioritariamente di persone preparate e con la voglia di dare il meglio per un sempre più attento servizio al paziente.
ACCADEMIA DI QUALITOLOGIA www.qualitologia.it qualitologia@qualitologia.it

\section{PROGETTO "COSTRUZIONE DI UN SISTEMA DI GESTIONE PER LA QUALITA' " IN UNA STRUTTURA SANITARIA}

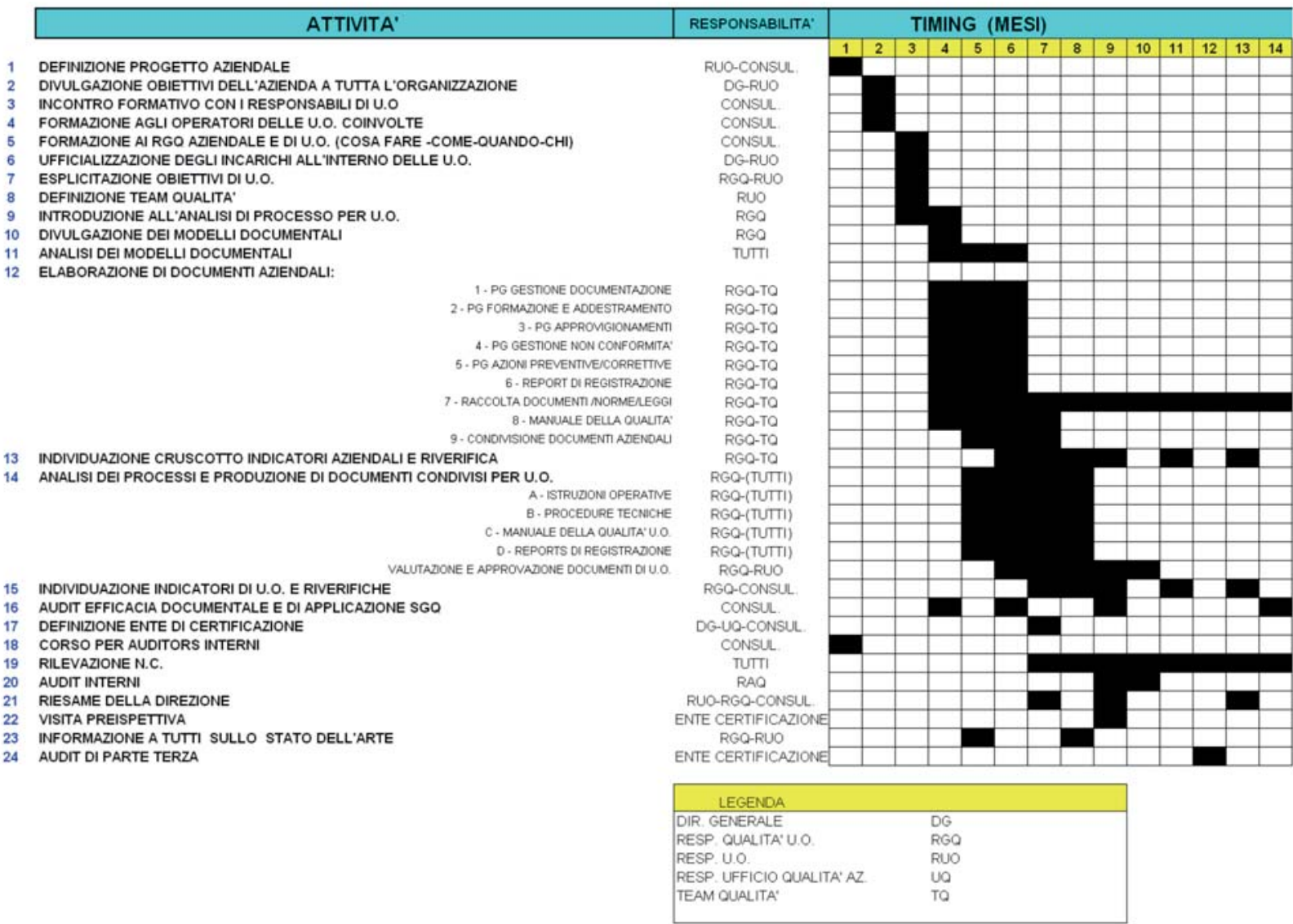

